\title{
Finite-Difference Methods for Nonlinear Hyperbolic Systems
}

\author{
By A. R. Gourlay and J. Ll. Morris*
}

Introduction. Finite-difference schemes of explicit and implicit type are derived for the numerical solution of first-order nonlinear hyperbolic systems, both in conservation and nonconservation form.

The discussion will be restricted to problems in one- and two-space dimensions, and to problems which have smooth solutions.

\section{PART I}

1. One-Space Dimension. Consider the first-order system of conservation laws

$$
\partial u / \partial t+\partial f / \partial x=0, \quad u(x, 0)=u_{0}(x), \quad t \geqq 0,
$$

where $f$ is a vector function of the components of $u$ and $u$ is an unknown vector function of $x, t$.

If the differentiation is carried out in (1.1) the equation

$$
\partial u / \partial t+A(u) \partial u / \partial x=0
$$

is obtained where $A(u)$ is the Jacobian matrix of the components of $f$ with respect to the components of $u$. Equation (1.2) is said to be hyperbolic if the eigenvalues of the matrix $\mu I+\theta A$ are real for all real numbers $\mu, \theta$.

Several authors have proposed finite-difference schemes for the numerical integration of (1.1) (or (1.2)). In [6], Lax and Wendroff introduced an explicit scheme which is stable if the Courant-Friedrichs-Lewy condition [2] is satisfied. In [10], Richtmyer showed how the Lax-Wendroff scheme could be written as a two-step process. Strang [13], has also considered the Lax-Wendroff scheme and in addition has examined the application of Runge-Kutta type methods to the integration of (1.1). Implicit methods, which are more difficult to apply, appear only to have been considered by Gary [4] although Richtmyer [10] has hinted at their possible use.

In Section 2 we will develop a general two-step process and in particular a new predictor-corrector scheme. In Section 3 an implicit scheme, similar in nature to Gary's scheme, will be considered.

2. Explicit One-Dimensional Case. We shall employ the following notation:

$$
\begin{aligned}
u(i h, m k) & \equiv u_{i}^{m} \equiv u_{m}, \\
H_{x} u_{m} & =u_{i+1}^{m}-u_{i-1}^{m}, \\
p & =k / h,
\end{aligned}
$$

where $h, k$ are the mesh spacings in the space and time directions, respectively, on the superimposed grid.

Received June 13, 1966. Revised January 13, 1967.

* Present address: Department of Mathematics, University of Dundee, Dundee, Scotland. 
In his review paper [10], Richtmyer formulates the explicit Lax-Wendroff method [6] as the two-step procedure

$$
\begin{aligned}
& u_{m+\frac{1}{2}}=\hat{u}_{m}-p / 4 H_{x} f_{m}, \\
& u_{m+1}=u_{m}-p / 2 H_{x} f_{m+\frac{1}{2}},
\end{aligned}
$$

where

$$
\hat{u}_{m}=\frac{1}{2}\left[u_{i+1}^{m}+u_{i-1}^{m}\right] .
$$

This procedure may be regarded as calculating the value $u_{m+1}$ by introducing an intermediate or auxiliary value $u_{m+\frac{1}{3}}$. This value is an approximation to the solution at the point $\left(i h,\left(m+\frac{1}{2}\right) k\right)$, but it is only correct to first-order, whereas the overall scheme (2.1) is correct to second-order. It is not obvious what is gained by restricting the intermediate value to be an approximation to the value $u_{m+\frac{3}{3}}$.

The scheme

$$
u_{m+1}^{*}=\hat{u}_{m}-a p H_{x} f_{m}
$$

provides an approximation to the value of $u$ at the point $(i h,(m+2 a) k)$, correct to first-order. Let us therefore consider a scheme which employs (2.2) as a first step and the general formula

$$
u_{m+1}=u_{m}-p H_{x}\left[b f_{m}+c f_{m+1}^{*}\right]
$$

where $b$ and $c$ are constants and $f_{m+1}^{*}=f\left(u_{m+1}^{*}\right)$ as the second step.

If we substitute for the starred values in (2.3) by means of formula (2.2) and expand the resulting difference scheme by Taylor's theorem, retaining terms up to and including those of order $h^{2}$, we obtain

$$
u_{m+1}=\left[u-2(b+c) k \frac{\partial f}{\partial x}+4 a c k^{2} \frac{\partial}{\partial x}\left(A \frac{\partial f}{\partial x}\right)\right]_{m}+O\left(h^{3}\right) .
$$

A Taylor expansion of $u_{m+1}$ in terms of $u_{m}$ and its derivatives yields

$$
u_{m+1}=\left[u+k \frac{\partial u}{\partial t}+\frac{k^{2}}{2} \frac{\partial^{2} u}{\partial t^{2}}+\cdots\right]_{m} .
$$

If we now use the relations (1.1) and

$$
\frac{\partial^{2} u}{\partial t^{2}}=-\frac{\partial^{2} f}{\partial x \partial t}=+\frac{\partial}{\partial x}\left(-\frac{\partial f}{\partial t}\right)=\frac{\partial}{\partial x}\left(-\frac{\partial f}{\partial u} \frac{\partial u}{\partial t}\right)=\frac{\partial}{\partial x}\left(A \frac{\partial f}{\partial x}\right)
$$

we have

$$
u_{m+1}=\left[u-k \frac{\partial f}{\partial x}+\frac{k^{2}}{2} \frac{\partial}{\partial x}\left(A \frac{\partial f}{\partial x}\right)\right]_{m}+O\left(h^{3}\right) .
$$

A comparison of (2.4) and (2.5) shows that in order that the general two-step method be accurate to order $h^{2}$, the equations

$$
2(b+c)=1, \quad 8 a c=1,
$$

must be satisfied. This set of equations has the solution

$$
b=\frac{1}{2}(1-1 / 4 a), \quad c=1 / 8 a
$$


in terms of the parameter a. Formulae (2.2) and (2.3) now form the two-step method

$$
\begin{aligned}
& u_{m+1}^{*}=\hat{u}_{m}-a p H_{x} f_{m}, \\
& u_{m+1}=u_{m}-p / 2 H_{x}\left[(1-1 / 4 a) f_{m}+f_{m+1}^{*} / 4 a\right] .
\end{aligned}
$$

The choice $a=\frac{1}{4}$ reduces (2.6) to the two-step Lax-Wendroff method (2.1).

Let us now examine the stability of the formula (2.6) with respect to growth of round-off errors. We assume that the stability of a nonlinear finite-difference scheme is governed by the local amplification matrix [13].

This is equivalent to considering the stability of (2.6) when the matrix $A$ is a constant. In this case no loss of generality occurs if we assume $A$ to be symmetric. (This assumption simplifies the stability analysis.) If formula (2.6) is linearized, the scheme

$$
\begin{aligned}
& u_{m+1}^{*}=\hat{u}_{m}-a p A H_{x} u_{m} \\
& u_{m+1}=u_{m}-p / 2 A H_{x}\left[\left(1-\frac{1}{4 a}\right) u_{m}+\frac{1}{4 a} u_{m+1}^{*}\right]
\end{aligned}
$$

is obtained. Elimination of $u_{m+1}^{*}$ from (2.7) leads to the formula

$$
u_{m+1}=u_{m}-\frac{p A}{2} H_{x}\left[u_{i}{ }^{m}+\frac{1}{8 a}\left(u_{i+1}^{m}-2 u_{i}{ }^{m}+u_{i-1}^{m}\right)\right]+\frac{p^{2} A^{2}}{8} H_{x}{ }^{2} u_{m} \text {. }
$$

If a Fourier decomposition of the errors is made in the usual manner, it follows that the amplification matrix $G$ of (2.8) (and hence locally of (2.6)) is given by

$$
G=I-\frac{1}{2} p^{2} A^{2} \sin ^{2} \beta h-p A i \sin \beta h\left[1+\frac{1}{4 a}(-1+\cos \beta h)\right]
$$

where $\beta$ is a real number. The Lax-Richtmyer condition for stability [8] requires that

$$
\|G * G\| \leqq 1
$$

This is satisfied if the eigenvalues of $G * G$ are less than one in modulus.

If the eigenvalues of $A$ are given by $|A-\lambda I|=0$, it is easily seen that the eigenvalues $g,(g \geqq 0)$, of $G * G$ are given by

$$
g=1-p^{2} \lambda^{2} \sin ^{2} \beta h\left[1-\frac{p^{2} \lambda^{2}}{4} \sin ^{2} \beta h-\left(1-\frac{1}{2 a} \sin ^{2} \frac{\beta h}{2}\right)^{2}\right]
$$

and therefore for stability we require

$$
1-\frac{p^{2} \lambda^{2}}{4} \sin ^{2} \beta h-\left(1-\frac{1}{2 a} \sin ^{2} \frac{\beta h}{2}\right)^{2} \geqq 0,
$$

that is,

$$
p^{2} \lambda^{2} \leqq\left(4 a-\sin ^{2} \beta h / 2\right) / 4 a^{2} \cos ^{2}(\beta h / 2)
$$

for all real $\beta$.

Since $p^{2} \lambda^{2} \geqq 0$, we have immediately that

$$
4 a-\sin ^{2}(\beta h / 2) \geqq 0
$$


for all real $\beta$. This requires that $a \geqq 1 / 4$. Let

$$
Q(z)=(4 a-1+z) / 4 a^{2} z, \quad 0 \leqq z \leqq 1,
$$

then

$$
d Q / d z=(1-4 a) / 4 a^{2} z^{2}
$$

Since $Q(z) \geqq 0$ and $a \geqq 1 / 4$, it follows that $d Q / d z \leqq 0$ and that the minimum of $Q(z)$ occurs at $z=1$.

It follows that for stability

$$
p^{2} \lambda^{2} \leqq \min _{0 \leqq \cos ^{2}(\beta h / 2) \leqq 1} \frac{4 a-1+\cos ^{2}(\beta h / 2)}{4 a^{2} \cos ^{2}(\beta h / 2)}=\frac{1}{a},
$$

that is,

$$
p|\lambda| \leqq 1 / \sqrt{ } a .
$$

Since the Courant-Friedrichs-Lewy condition [2] requires that $p|\lambda| \leqq 2$, it follows that we have "maximum" stability if $a=\frac{1}{4}$ and conditional stability $p|\lambda| \leqq 1 / \sqrt{ } a$ otherwise.

In an actual implementation, the above schemes require data not only on the initial line $t=0$ but also on the two bounding lines $x=x_{0}, x_{1}$, say. The experiments of Richtmyer and Morton [11], Gary [4], and Parter [9] show that the incorporation of such data must be treated with care. Whilst a procedure for smoothly incorporating the boundary data can readily be derived, we propose to use the following technique. We shall regard (2.6) as generating the predictor-corrector scheme

$$
\begin{aligned}
u_{m+1}^{*} & =\hat{u}_{m}-p a H_{x} f_{m}, \\
u_{m+1}^{(j+1)} & =u_{m}-p / 2 H_{x}\left[\left(1-\frac{1}{4 a}\right) f_{m}+\frac{1}{4 a} f_{m+1}^{(j)}\right],
\end{aligned}
$$

for $j=1, \cdots$ where

$$
f_{m+1}^{(1)}=f_{m+1}^{*}
$$

and the sequence of iterates

$$
\left\{u_{m+1}^{(j)}, j=2, \cdots\right\}
$$

is required to tend to the solution at $u(i h,(m+1) k)$.

We are therefore really finding the solution of the replacement

$$
u_{m+1}=u_{m}-p / 2 H_{x}\left[\left(1-\frac{1}{4 a}\right) f_{m}+\frac{1}{4 a} f_{m+1}\right]
$$

by an iterative procedure. If the expression on the right-hand side of (2.10) is expanded by Taylor's theorem in terms of $u_{m}$ and its time derivatives to order $k^{2}$, the expansion

$$
u_{m+1}=\left[u+k \frac{\partial u}{\partial t}+\frac{k^{2}}{4 a} \frac{\partial^{2} u}{\partial t^{2}}\right]_{m}+O\left(k^{3}\right)
$$

is obtained where use has been made of (1.1). Whilst every replacement is a consistent one, only the choice $a=\frac{1}{2}$ gives a corrector which may converge to the 
order of the overall scheme (2.9). Let us now demonstrate the convergence of the iterated corrector in (2.9).

Subtracting (2.10) from the corrector in (2.9) gives the equation

$$
u_{m+1}^{(j+1)}-u_{m+1}=-p H_{x}\left[f_{m+1}^{(j)}-f_{m+1}\right] / 8 a .
$$

To carry the analysis further we are again forced to linearize this equation and obtain

$$
u_{m+1}^{(j+1)}-u_{m+1}=-p A H_{x}\left[u_{m+1}^{(j)}-u_{m+1}\right] / 8 a,
$$

where we now assume that $A$ is constant at any time level. If the notation

$$
\epsilon_{i}^{j+1}=u_{m+1}^{(j+1)}-u_{m+1}, \quad E_{j+1}=\left(\epsilon_{1}^{j+1} \cdots \epsilon_{N}^{j+1}\right)
$$

is introduced, then the totality of equations of the form (2.12) for consecutive iterates may be written in the form

$$
E_{j+1}=A_{1} E_{j}
$$

where $A_{1}$ is block tridiagonal (with zeroes on the diagonal) and where we have assumed periodic boundary conditions. The iterative process will therefore converge if $\left\|A_{1}\right\|<1$. The analysis given by Gary [4, p. 16] to prove the convergence of his explicit iterative scheme may then be applied without change. We shall not repeat it but merely state the conclusion reached; namely, that for convergence we require $p|\lambda|<4 a$.

The most satisfactory predictor-corrector process is obtained when $a=\frac{1}{2}$, for only in this case do the iterates converge to the order of the overall method. This scheme is given by

$$
\begin{aligned}
u_{m+1}^{(1)} & =\hat{u}_{m}-p / 2 H_{x} f_{m}, \\
u_{m+1}^{(j+1)} & =u_{m}-p / 4 H_{x}\left[f_{m}+f_{m+1}^{(j)}\right], \quad(j=1,2,3, \cdots) .
\end{aligned}
$$

Let us briefly summarize two interesting points arising from the above analysis.

(a) For overall stability the condition is $a \geqq 1 / 4$, implying that the prediction must not be made in the interval between the points $u(i h, m k)$ and $u\left(i h,\left(m+\frac{1}{2}\right) k\right)$.

(b) We may in fact use a prediction in advance of the $(m+1)$ th level and still maintain stability. For example, if $a=1$ the prediction is at the $(m+2)$ th level and the appropriate formulae are then

$$
\begin{aligned}
& u_{m+1}^{*}=\hat{u}_{m}-p H_{x} f_{m}, \\
& u_{m+1}=u_{m}-p / 8 H_{x}\left[3 f_{m}+f_{m+1}^{*}\right] .
\end{aligned}
$$

Although all the previous analysis has been carried out for the case of a system in conservation form, it may easily be verified that if the equation is not in conservation form, but of the type

$$
\partial u / \partial t+A(u) \partial u / \partial x=0,
$$

then a similar general scheme may be obtained. It now takes the form

$$
\begin{aligned}
& u_{m+1}^{*}=\hat{u}_{m}-p a A_{m} H_{2} u_{m} \\
& u_{m+1}=u_{m}-p / 2\left[(1-1 / 4 a) A_{m} H_{2} u_{m}+(1 / 4 a) A_{m+1}^{*} H_{x} u_{m+1}^{*}\right]
\end{aligned}
$$


where $A_{m} \equiv A\left(u_{m}\right)$ and $A_{m+1}^{*} \equiv A\left(u_{m+1}^{*}\right)$.

The above schemes bear a strong resemblance to several finite-difference methods for solving the ordinary differential equation $y^{\prime}=f(x, y)$. For example, the Lax-Wendroff method (2.1) is similar to the "improved Euler method,"

$$
\begin{aligned}
y_{n+1 / 2} & =y_{n}+\frac{h}{2} f_{n}, \quad f_{n}=f\left(x_{n}, y_{n}\right), \\
y_{n+1} & =y_{n}+h f_{n+1 / 2},
\end{aligned}
$$

whereas the scheme (2.14) mirrors the "improved Euler-Cauchy method,"

$$
y_{n+1}^{*}=y_{n}+h f_{n}, \quad y_{n+1}=y_{n}+\frac{1}{2} h\left[f_{n}+f_{n+1}^{*}\right] \text {. }
$$

3. Implicit One-Dimensional Scheme. In [4], Gary considered the following scheme

$$
\begin{aligned}
& u_{m+1}^{*}=u_{m}-p / 2 A_{m} H_{x} u_{m}, \\
& u_{m+1}=u_{m}-p / 4 A\left(\frac{1}{2}\left[u_{m}+u_{m+1}^{*}\right]\right) H_{x}\left(u_{m+1}+u_{m}\right),
\end{aligned}
$$

for the numerical integration of the system of equations in nonconservation form given by

$$
\partial u / \partial t+A(u) \partial u / \partial x=0 .
$$

This method is an approximation to the Crank-Nicolson scheme

$$
u_{m+1}=u_{m}-p / 4\left[A_{m} H_{x} u_{m}+A_{m+1} H_{x} u_{m+1}\right]
$$

and is again accurate to order $h^{2}$. It is easy to see that as it stands (3.2) cannot be solved for $u_{m+1}$ because of the nonlinear term $A\left(u_{m+1}\right)$.

The predictor used in (3.1) is in fact an unstable one. We prefer to use

$$
u_{m+1}=\hat{u}_{m}-p / 2 A_{m} H_{x} u_{m}
$$

and then apply (3.2) in the form

$$
u_{m+1}=u_{m}-p / 4\left[A_{m} H_{x} u_{m}+A_{m+1}^{*} H_{x} u_{m+1}\right] .
$$

This scheme is still accurate to order $h^{2}$ but now permits the calculation of $u_{m+1}$ by a block tridiagonal inversion. Methods for inverting such systems are well known [12], [14], and will not be repeated here. By linearizing (3.4) we may show that, like Gary's scheme, it has unconditional stability.

Whereas Gary's scheme was designed specifically for schemes in nonconservation form, it is easy to verify that the formulation of (3.4) for conservative systems is given by

$$
\begin{aligned}
& u_{m+1}^{*}=\hat{u}_{m}-p / 2 H_{x} f_{m}, \\
& u_{m+1}=u_{m}-p / 4 H_{x}\left[f_{m}+\widetilde{A}_{m+1}^{*} u_{m+1}\right],
\end{aligned}
$$

where $\tilde{A}(u)$ is defined by the relation $f(u)=\tilde{A}(u) \cdot u$. The formula (3.5) is again accurate to order $h^{2}$ and is unconditionally stable. The solution $u_{m+1}$ may be obtained once again by a block tridiagonal inversion.

Each equation occurring in (3.5) and (2.6) is in conservation difference form (Lax and Wendroff [6]). 

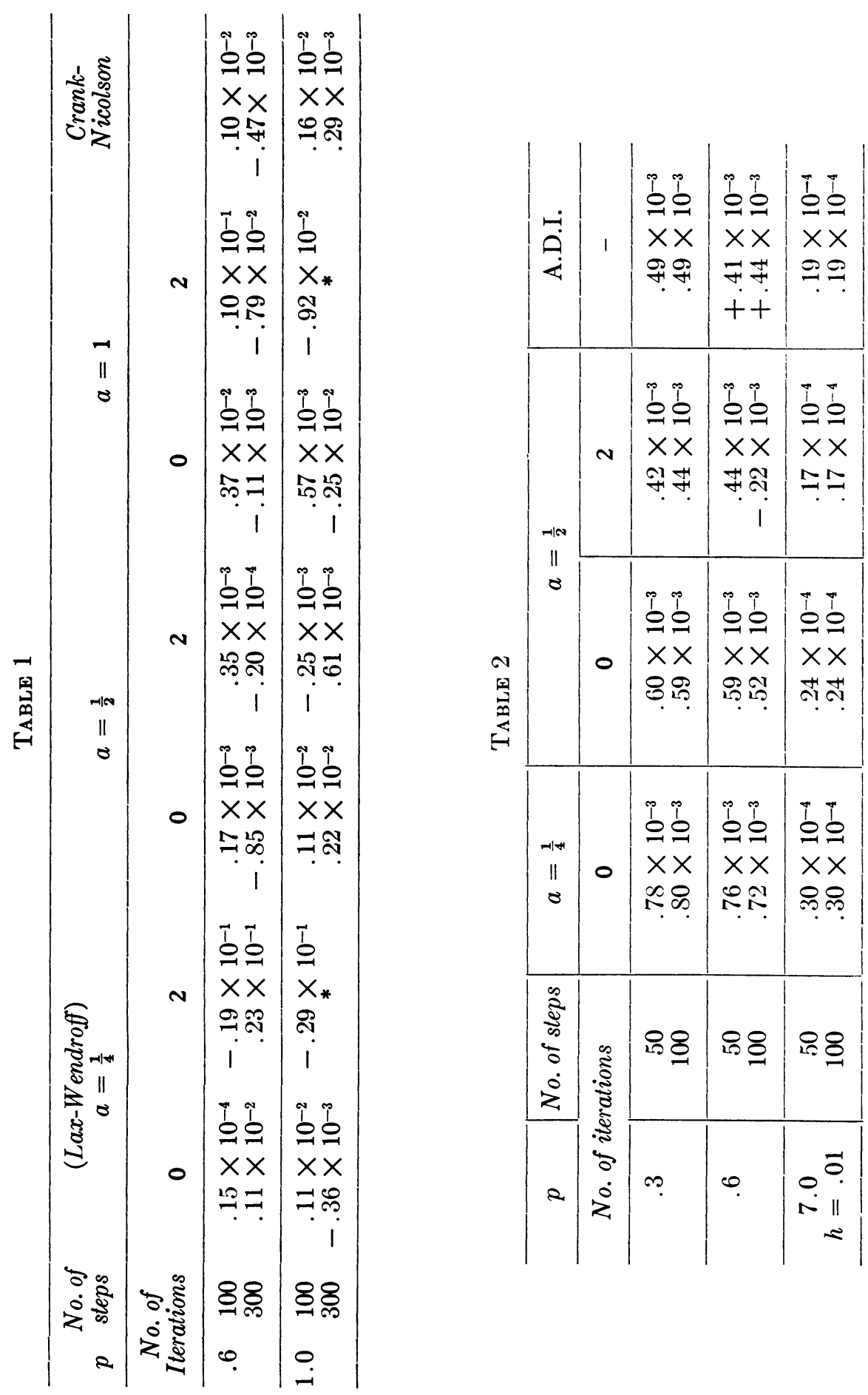
4. Numerical Results. In order to compare the above methods, several calculations were carried out for the simple problem

$$
\partial u / \partial t+\partial / \partial x\left(\frac{1}{2} u^{2}\right)=0, \quad u(x, 0)=x, \quad 0 \leqq x \leqq 1,
$$

which has the solution $u(x, t)=x /(1+t)$.

The values $a=\frac{1}{4}, \frac{1}{2}, 1$ were taken for the parameter in the scheme (2.6) and the iterated scheme (2.9). A comparison was then made between the results obtained from the schemes and the implicit scheme (3.5). In the calculations the values $h=0.1, p=0.6,1.0$ were taken and the results for the errors (the differences between the theoretical solution and the computed solution) are quoted in Table 1 for the point $x=\frac{1}{2}$.

The entries * in the table indicate that nonlinear instability had developed. Although this topic is of great importance we shall not deal with it here but merely note its occurrence. The results in the table tend to confirm the analysis given above, in that iteration in the case $a=\frac{1}{2}$ gives improved results, whereas iteration in the cases $a=\frac{1}{4}, 1$ leads to a loss in accuracy, and perhaps to nonlinear instability. The results quoted are representative of the calculations made with the various schemes.

It was found that no more than two iterations were required for convergence of the significant figures for the scheme $a=\frac{1}{2}$.

\section{PART II}

Two-Space Dimensions. We shall now extend the methods introduced in Part I to the case of the two-space dimensional system of conservation laws

$$
\partial u / \partial t+\partial f / \partial x+\partial g / \partial y=0
$$

or the nonconservative system

$$
\partial u / \partial t+A(u) \partial u / \partial x+B(u) \partial u / \partial y=0 .
$$

We shall assume that $A$ and $B$ may be simultaneously symmetrized in order to guarantee the hyperbolicity of the above system.

Explicit difference schemes have been proposed by Lax and Wendroff [7], Richtmyer [10], Strang [13], and Burstein [1] for the solution of these equations.

5. Explicit Two-Dimensional Case. In [10], Richtmyer shows that the two-step formulation of the Lax-Wendroff method for systems in two-space dimensions, is given by

$$
\begin{aligned}
& u_{m+1}^{*}=\hat{u}_{m}-p / 4\left[H_{x} f_{m}+H_{y} g_{m}\right], \\
& u_{m+1}=u_{m}-p / 2\left[H_{x} f_{m+1}^{*}+H_{y} g_{m+1}^{*}\right],
\end{aligned}
$$

where $u_{i, j}^{m} \equiv u_{m} \equiv u(i h, j h, m k)$

$$
\hat{u}_{m}=\frac{1}{4}\left[u_{i+1, j}^{m}+u_{i-1, j}^{m}+u_{i, j+1}^{m}+u_{i, j-1}^{m}\right]
$$

and the operators $H_{x}$ and $H_{y}$ are defined by 


$$
\begin{aligned}
& H_{x} u_{m}=u_{i+1, j}^{m}-u_{i-1, j}^{m}, \\
& H_{y} u_{m}=u_{i, j+1}^{m}-u_{i, j-1}^{m} .
\end{aligned}
$$

In exactly the same way as before we may generalize this scheme to the form

$$
\begin{aligned}
& u_{m+1}^{*}=\hat{u}_{m}-p a\left[H_{x} f_{m}+H_{y} g_{m}\right], \\
& u_{m+1}=u_{m}-p / 2\left[\left(1-\frac{1}{4 a}\right)\left[H_{x} f_{m}+H_{\nu} g_{m}\right]+\frac{1}{4 a}\left[H_{x} f_{m+1}^{*}+H_{y} g_{m+1}^{*}\right]\right],
\end{aligned}
$$

where once again we have second-order accuracy.

In order to examine the stability of the class (5.2), the equations are linearized and $u_{m+1}^{*}$ is eliminated. This produces the scheme

$$
u_{m+1}=u_{m}-p / 2\left[A H_{x}+B H_{y}\right]\left[\left(1-\frac{1}{4 a}\right) u_{m}+\frac{1}{4 a} \hat{u}_{m}\right]+\frac{p^{2}}{8}\left[A H_{x}+B H_{y}\right]^{2} u_{m},
$$

where $A, B$ are now assumed (as in the one-dimensional case) to be constant, symmetric matrices. After making the usual Fourier transform of the space variables, the amplification matrix $G$ is given by

$$
\begin{aligned}
G= & I-i p[A \sin \alpha h+B \sin \beta h]\left[\left(1-\frac{1}{4 a}\right)+\frac{1}{8 a}(\cos \alpha h+\cos \beta h)\right] \\
& -\frac{p^{2}}{2}[A \sin \alpha h+B \sin \beta h]^{2}
\end{aligned}
$$

where $\alpha, \beta$ are real numbers.

Let $Z$ denote the symmetric matrix $A \sin \alpha h+B \sin \beta h$. Then if $\lambda_{z}$ is an eigenvalue of $Z$, the eigenvalues of $G * G$ are given by

$$
g=\left(1-\frac{p^{2} \lambda_{Z}{ }^{2}}{2}\right)^{2}+p^{2} \lambda_{z}{ }^{2}\left[\left(1-\frac{1}{4 a}\right)+\frac{1}{8 a}(\cos \alpha h+\cos \beta h)\right]^{2} .
$$

For Lax-Richtmyer stability we require $g \leqq 1$, and therefore

$$
\frac{p^{2} \lambda_{Z}^{2}}{4} \leqq 1-\left[\left(1-\frac{1}{4 a}\right)+\frac{1}{8 a}(\cos \alpha h+\cos \beta h)\right]^{2}
$$

for all $\alpha, \beta$; that is

$$
p^{2} \leqq \min _{\alpha, \beta}\left\{\frac{1}{\lambda_{Z}{ }^{2}}\left[4-\frac{1}{16 a^{2}}[8 a-2+(\cos \alpha h+\cos \beta h)]^{2}\right]\right\} .
$$

Because of the dependence of $\lambda_{z}$ on $\alpha, \beta, A, B$ it will be necessary to consider the stability of the scheme (5.2) with regard to a specific problem. Following Richtmyer [10] we consider the equations of gas flow. There the maximum eigenvalue of the matrix $Z^{2}$ is given by

$$
(|q|+c)^{2}\left(\sin ^{2} \beta h+\sin ^{2} \alpha h\right)
$$

where $|q|$ is the speed of the fluid and $c$ is the local speed of sound. In this case, therefore, we have the stability condition

$$
p^{2}(|q|+c)^{2} \leqq \min _{\alpha, \beta}\left\{\frac{4-\frac{1}{16 a^{2}}[8 a-2+(\cos \alpha h+\cos \beta h)]^{2}}{\sin ^{2} \alpha h+\sin ^{2} \beta h}\right\}=M(a) .
$$


By analytical means, or by a simple scanning run on a computer it may be shown that $M(a)$ is negative for $a<\frac{1}{4}$ and that it has a maximum at $a=\frac{1}{4}$. Moreover, it decreases steadily in the range $\frac{1}{4} \leqq a \leqq \infty$. A graph showing the behaviour is given below in Fig. 1 .

Moreover, from (5.3) it can readily be seen that $a \geqq \frac{1}{4}$, independent of $\lambda_{z}$, is a necessary condition for stability.

It appears, therefore, that the Lax-Wendroff method $\left(a=\frac{1}{4}\right)$ is optimum for this class as far as stability is concerned.

However, we may still consider the case $a=\frac{1}{2}$ to see if the iteration of the corrector improves our accuracy or not. This scheme is given by

$$
\begin{aligned}
& u_{m+1}^{(1)}=\hat{u}_{m}-p / 2\left[H_{x} f_{m}+H_{y} g_{m}\right], \\
& u_{m+1}^{(j+1)}=u_{m}-p / 4\left[H_{x}\left(f_{m}+f_{m+1}^{(j)}\right)+H_{y}\left(g_{m}+g_{m+1}^{(j)}\right)\right], \quad(j=1,2,3, \cdots)
\end{aligned}
$$

and its stability range is $1 / \sqrt{ } 2$ times that of the Lax-Wendroff method. As before, we may derive the error equation in the form

$$
u_{m+1}^{(j+1)}-u_{m+1}=-p / 4\left[H_{x}\left(f_{m+1}^{(j)}-f_{m+1}\right)+H_{y}\left(g_{m+1}^{(j)}-g_{m+1}\right)\right]
$$

which on linearizing may be put in the form

$$
\epsilon^{j+1}=-p / 4\left[A H_{x} \epsilon^{j}+B H_{y} \epsilon^{j}\right]
$$

where $\epsilon^{j+1}=u_{m+1}^{(j+1)}-u_{m+1}$.

The application of an analysis exactly similar to the one-dimensional case shows that convergence will occur if

$$
p\left|\lambda_{A}\right| \leqq 1, \quad p\left|\lambda_{B}\right| \leqq 1
$$

which are satisfied by the stability requirement.

Just as in the one-dimensional case, we can show that the above schemes may. be derived for the equations in nonconservation form. Thus (5.2) becomes

$$
\begin{aligned}
& u_{m+1}^{*}=\hat{u}_{m}-p a\left[A_{m} H_{x}+B_{m} H_{y}\right] u_{m} \\
& u_{m+1}=u_{m}-p / 2\left[\left(1-\frac{1}{4 a}\right)\left(A_{m} H_{x}+B_{m} H_{y}\right) u_{m}+\frac{1}{4 a}\left(A_{m+1}^{*} H_{x}+B_{m+1}^{*} H_{y}\right) u_{m+1}^{*}\right]
\end{aligned}
$$

again accurate to order $h^{2}$.

6. Implicit Two-Dimensional Cases. The natural extension of the CrankNicolson type scheme is the nonlinear analogue of the alternating direction implicit (A.D.I.) method considered by Gourlay and Mitchell [5]. This scheme

$$
\begin{aligned}
& {\left[u_{m+1}+p / 4\left[H_{x} f_{m+1}+H_{y} g_{m+1}\right]+p^{2} / 16 H_{y} \widetilde{B}_{m+1} H_{x} f_{m+1}\right]} \\
& =\left[u_{m}-p / 4\left(H_{x} f_{m}+H_{y} g_{m}\right)+p^{2} / 16 H_{y} \widetilde{B}_{m} H_{x} f_{m}\right]
\end{aligned}
$$

is second-order correct and is unconditionally stable in the linearized sense. This scheme involves nine points at each of two neighbouring time levels. To solve this implicit scheme we introduce a predictor-corrector method which employs a $\mathrm{D}^{\prime}$ jakonov [3] type factorization of scheme (6.1). We therefore obtain the A.D.I. method

$$
u_{m+1}^{* *}=\hat{u}_{m}-p / 2\left[H_{x} f_{m}+H_{y} g_{m}\right]
$$


(6.3)

$$
\left[I+p / 4 H_{y} \tilde{B}\left(u_{m+1}^{* *}\right)\right] u_{m+1}^{*}=Q, \quad\left[I+p / 4 H_{x} \tilde{A}\left(u_{m+1}^{* *}\right)\right] u_{m+1}=u_{m+1}^{*},
$$

where $\widetilde{A}(u), \widetilde{B}(u)$ are defined by the notations

$$
f(u)=\widetilde{A}(u) u, \quad g(u)=\widetilde{B}(u) u
$$

and where $Q$ is the term on the right-hand side of $(6.1)$.

The scheme ((6.2) and (6.3)) obtains $u_{m+1}$ by two block tridiagonal inversions and takes roughly twice as long to run on a computer as the explicit method. The method is still accurate to order $h^{2}$.

Also, we may derive an A.D.I. method for the case of a system in nonconservation form. This is given by

$$
\begin{aligned}
u_{m+1}^{* *} & =\hat{u}_{m}-p / 2\left[A_{m} H_{x}+B_{m} H_{y}\right] u_{m}, \\
{\left[I+p / 4 B_{m+1}^{* *} H_{y}\right] u_{m+1}^{*} } & =\left[I-p / 4\left[A_{m} H_{x}+B_{m} H_{y}\right]+p^{2} / 16 B_{m} H_{y} A_{m} H_{x}\right] u_{m}, \\
{\left[I+p / 4 A_{m+1}^{* *} H_{x}\right] u_{m+1} } & =u_{m+1}^{*} .
\end{aligned}
$$

7. Numerical Results. To compare the methods in the two-space dimensional case the problem

$$
\frac{\partial u}{\partial t}+\frac{\partial}{\partial x}\left(\frac{1}{4} u^{2}\right)+\frac{\partial}{\partial y}\left(\frac{1}{4} u^{2}\right)=0, \quad u(x, y, 0)=\frac{1}{4}(x+y)^{2},
$$

was chosen. This has the theoretical solution

$$
u(x, y, t)=\left[\frac{1-\{1+(x+y) t\}^{1 / 2}}{t}\right]^{2}, \quad t>0 .
$$

Once again the region $0 \leqq x, y \leqq 1$ was considered and a square grid with spacing $h=0.1$ was superimposed on the region. The methods were run for varying $p$ and the results are quoted in Table 2 for the scheme (5.2) with $a=\frac{1}{4}, \frac{1}{2}$ and the A.D.I. method, the entries in the table are for the errors at the point $\left(\frac{1}{2}, \frac{1}{2}\right)$.

It may be noticed that iterating the corrector at $a=\frac{1}{2}$ does appear to improve the accuracy. Once again, only two iterations are required. If the corrector is iterated for values of $a \neq \frac{1}{2}$, the divergence was rapid and nonlinear instability again developed.

In fact, very little can be said as to which scheme is more accurate. Although the above results are representative, it has been noticed that the A.D.I. method is more accurate at boundary planes than in the centre of the region, whereas the explicit methods are more accurate in the centre of the region. The stability of the explicit methods when $p=7.0$ is certainly surprising.

Conclusions. Several methods have been proposed for the numerical integration of systems of conservation laws. In one-space dimension, it would appear that the new predictor-corrector scheme has much to offer, as does the Crank-Nicolson scheme. In two-space dimensions the same advantage is gained over the existing Lax-Wendroff scheme.

It would obviously be desirable to test these methods on physical problems involving shocks or discontinuities. It is hoped to carry out this investigation in the future. 
The methods developed in the paper can obviously be extended in a natural way to a higher number of space dimensions.

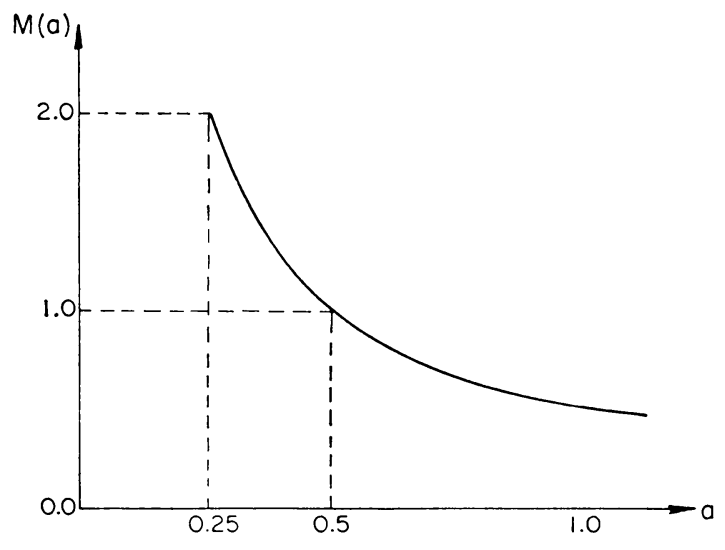

Figure 1

Acknowledgements. Mr. A. R. Gourlay's share of the work was carried out whilst he was in receipt of a Carnegie Research Scholarship. Mr. J. Ll. Morris's share of the work was carried out under a grant from the Science Research Council.

Department of Applied Mathematics

University of St. Andrews

St. Andrews, Scotland

1. S. Z. Burstein, Numerical Calculations of Multidimensional Shocked Flows, N.Y.U. Courant Inst. Math. Sci. Res. Dep. N.Y.O.-10, 433, 1963.

2. R. Courant, K. O. Friedrichs \& H. Lewy, On the Partial Difference Equations of Mathematical Physics, translated from German by Phyllis Fox, N.Y.U. Courant Inst. Math. Sci. Res. Dep. N.Y.O.-7689, 1956.

3. G. YE. D'JAKONOv, "Difference schemes with a disintegrating operator for multi-dimensional problems," translated from the Russian, U.S.S.R. Comp. Math. and Math. Physics, v. 4, 1964 , pp. $581-607$.

4. J. GARY, "On certain finite difference schemes for hyperbolic systems," Math. Comp., v. 18, 1964, pp. 1-18. MR $28 \# 1776$.

5. A. R. Gourlay \& A. R. Mitchell, "A stable implicit difference method for hyperbolic systems in two space variables," Numer. Math., v. 8, 1966, pp. 367-375. MR 33 \#6850.

6. P. D. Lax \& B. Wendroff, "Systems of conservation laws," Comm. Pure Appl. Math., v. 13,1960 , pp. $217-237$. MR $22 \# 11523$.

7. P. D. LAX \& B. WENDROFF, "Difference schemes for hyperbolic equations with high order of accuracy," Comm. Pure Appl. Math., v. 17, 1964, pp. 381-398. MR 30 \#22.

8. P. D. LAX \& R. D. RichtMYER, "Survey of the stability of linear finite difference equations," Comm. Pure Appl. Math., v. 9, 1956, pp. 267-293. MR 18, 48.

9. S. V. PARTER, "Stability, convergence and pseudo-stability of finite-difference equations for an over-determined problem," Numer. Math., v. 4, 1962, pp. 277-292. MR 26 \#5740.

10. R. D. Richtmyer, $A$ Survey of Difierence Methods for Non-steady fluid Dymamics, N.C.A.R. Tech. Note 63-2, 1963.

11. R. D. Richtmyer \& K. W. Morton, Stability Studies for Finite Difference Equations, N.Y.U. Courant Inst. Math. Sci. Res. Dep. N.Y.O.-1480-5, 1964.

12. S. Schechter, "Quasi-tridiagonal matrices and type-insensitive difference equations," Quart. Appl. Math., v. 18, 1960-61, pp. 258-295. MR 22 \#133.

13. W. G. Strang, "Accurate partial difference methods. II. Non-linear problems," Numer. Math., v. 6, 1964, pp. 37-46. MR $29 \# 4215$.

14. R. S. VArga, Matrix Iterative Analysis, Prentice-Hall, Englewood Cliffs, N. J., 1962. MR 28 \#1725. 